\title{
Vibrations of Elastically Restrained Circular Plates Resting on Partial Winkler Foundation
}

\author{
Chellapilla Kameswara Rao,"1 and Lokavarapu Bhaskara Rao ${ }^{2}$ \\ ${ }^{1}$ Hitech College of Engineering \& Technology, Gandipet-Himayatnagar, Hydreabad-500075, India \\ ${ }^{2}$ Deparment of Mechanical Engineering, D.R.K. College of Engineering and Technology, Hyderabad, Andhra Pradesh, \\ India
}

\begin{abstract}
This work describes a study of vibration characteristics of thin circular plates with elastic edge support and resting on partial Winkler-type elastic foundation. The foundation is described by the Winkler model, which is called as single parameter foundation. The exact analytical method is used to derive the frequency equation of the circular plate with elastic edge support-conditions and resting on partial elastic foundation system. Parametric investigations on the behavior of circular plates with elastic edge support and resting on partial elastic foundation have been carried out with respect to various values of transverse stiffness parameter, foundation parameter for a variety of boundary conditions. Extensive data is tabulated so that pertinent conclusions can be arrived at on the influence of translational edge restraint, and the foundation modulus parameters of the Winkler foundation on the natural frequencies of uniform isotropic circular plates. A comparison of the results obtained here in this paper with those available in the literature shows an excellent agreement.
\end{abstract}

Keywords: Circular plate, restrained edge, translational spring stiffness, elastic foundation.

\section{INTRODUCTION}

The structural behavior of circular plates on elastic foundation is of great interest for the design on many engineering problems. Such plate systems can be found in many engineering applications, ranging from more conventional civil engineering, mechanical engineering and marine engineering to an aerospace engineering. Research work in this area has been discussed in a series of papers by Lessia [1,2] and Bert [3, 4]. The vibration of a circular plate supported laterally by an elastic foundation was studied by Leissa in Ref. [5] from which he deduced that the effect of a Winkler foundation merely increases the square of the natural frequency of the plate by a constant. Laura et al. [6], while studying the case of a circular plate partially embedded in a Winkler foundation, found that a simple frequency relation like the above no longer holds good, and thus reached a similar conclusion. The most general soil model used in practical applications is the Winkler model [7] in which the soil layer is represented by unconnected closely spaced elastic springs.

The present study of circular plates on elastic foundations with elastic edge support finds useful applications in foundation designs of large storage tanks, deep-sea pressure vessels and heavy machines [8].

However, studies of the vibration of plates considering the combined effects of elastic foundations and elastic

*Address correspondence to this author at the Hitech College of Engineering \& Technology, Gandipet-Himayatnagar, Hydreabad-500075, India; E-mail: chellapilla95@gmail.com constraints are relatively scarce in the literature [9]. The vibration characteristics of plates resting on an elastic medium are different from those of the plates supported only on the boundary. There are many difficulties which very often arise due to complexity and uncertainty of boundary conditions. This uncertainty could be due to practical engineering applications where the edge of the plate does not fall into the classical boundary conditions. The accepted fact is that the condition on a periphery often tends to be part away between the classical boundary conditions (simply supported, free, pinned) and non-classical boundary conditions (elastic edge restraints) [10]. Therefore, when the boundary conditions of the plate deviate from classical cases, elastic edge restraints need to be considered. The present study considers the problem of vibrations of circular plates elastically restrained against translation and resting on partial elastic foundation i.e. on partial Winkler foundation. In this paper, exact solutions for first Eigen-frequencies of thin circular plates for various values of non-dimensional parameters are presented in graphical and tabular form, which may be useful for engineers in practice as well as to researchers as benchmark results for checking the relative accuracy of the approximate results obtained through alternate methods of solution.

\section{DEFINITION OF THE PROBLEM}

Consider a thin isotropic, circular plate of radius $R$, uniform thickness $h$, Young's modulus $E$, flexural rigidity $D$ and Poisson's ratio $v$ as shown in Fig. (1). The plate edge is considered to be elastically restrained in translation and partially supported on Winkler foundation. The plate is also assumed to be made of linearly elastic, homogeneous and isotropic material. However, the effects of shear 
deformation and rotary inertia are neglected in the present paper as the plate considered is quite thin. The problem at hand is to determine the frequencies of a circular plate with elastically restrained edge and resting on partial elastic foundation.

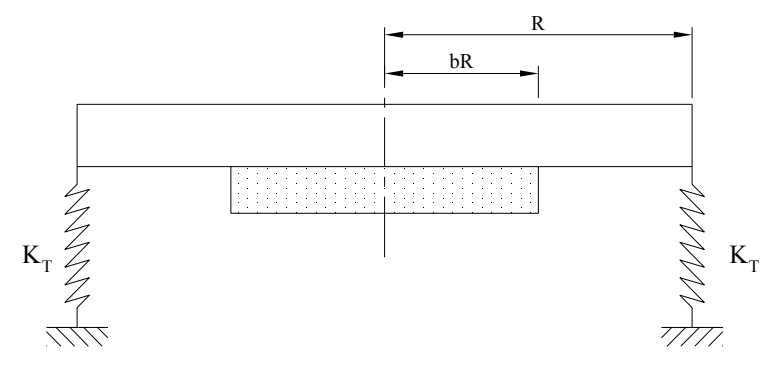

Fig. (1). A thin circular plate with rotational $K_{R}$ and translational $\mathrm{K}_{\mathrm{T}}$ elastic edge constraints and supported on partial elastic foundation.

\section{MATHEMATICAL FORMULATION}

The geometrical and loading configuration of the plate is axi-symmetric and consequently, deflection shape of the plate will be axially symmetric as well. Consider a circular plate of radius $R$ being supported in the interior by a foundation of radius $b R$ as shown in Fig. (1). Let the subscript I denote the outer region $b \leq \bar{r} \leq 1$ and the subscript II denote the inner region $0 \leq \bar{r} \leq b$. Here, all lengths are normalized with respect to $R$. The nondimensional radius at the outer edge is 1 and at the inner edge is b. As per the classical Kirchhoff's plate theory [11, 12], the fourth order differential equation describing the free flexural vibrations of a thin circular uniform plate for region I, in polar coordinates $(r, \theta)$ is given by:

$\nabla^{4} w_{I}-k^{4} w_{I}=0$

where $k^{4}=R^{4} \omega^{2} \rho / D$ which is the non-dimensional frequency parameter.

The plate Eq. (5) for region II is:

$\nabla^{4} w_{I I}-k^{4} w_{I I}+\lambda^{4} w_{I I}=0$

where $\lambda^{4}=R^{4} K_{w} / D$ which represents the no-dimensional foundation stiffness parameter.

Let the solution to the Eq. (1) be represented as:

$w=u(r) \cos (n \theta)$

where $r$ is the radius normalized w.r.t $R, n$ is the number of nodal diameters. The function $u$ a linear combination of the Bessel functions $J_{n}(k r), Y_{n}(k r), I_{n}(k r) \& K_{n}(k r)$ is:

$u_{I}(r)=C_{1} J_{n}(k r)+C_{2} Y_{n}(k r)+C_{3} I_{n}(k r)+C_{4} K_{n}(k r)$

where $C_{1}, C_{2}, C_{3} \& C_{4}$ are constants.

$J_{n}($.$) and Y_{n}($.$) are the Bessel functions of the first and second$ kinds of order $n$ respectively.

$I_{n}(.) \operatorname{and} K_{n}($.$) are the modified Bessel functions of the first$ and second kinds of order $n$ respectively.

Substituting Eq. (4) in Eq. (3) gives the following:
$w_{I}(r, \theta)=\left[\begin{array}{l}C_{1} J_{n}(k r)+C_{2} Y_{n}(k r)+ \\ C_{3} I_{n}(k r)+C_{4} K_{n}(k r)\end{array}\right] \cos (n \theta)$

Unlike Eq. (1), the general solution to Eq. (2), is more complicated and the following three special cases are considered in this paper.

Case (i) If $k \succ \lambda$, the solution to Eq. (2) is:

$u_{I I}(r)=C_{5} J_{n}\left(k_{1} r\right)+C_{6} I_{n}\left(k_{1} r\right)$

where $k_{1}=\left(k^{4}-\lambda^{4}\right)^{\frac{1}{4}}$.

Substituting Eq. (6) in Eq. (3), we get:

$w_{I I}(r, \theta)=\left[C_{5} J_{n}\left(k_{1} r\right)+C_{6} I_{n}\left(k_{1} r\right)\right] \cos (n \theta)$

Case (ii) If $k=\lambda$, the solution to Eq. (2) is given by:

$u_{I I}(r)=C_{5} r^{n}+C_{6} r^{n+2}$

Substitution of Eq. (8) in Eq. (3) gives the following:

$w_{I I}(r, \theta)=\left[C_{5} r^{n}+C_{6} r^{n+2}\right] \cos (n \theta)$

Case (iii) If $k<\lambda$, the solution to Eq. (2) is given by:

$u_{I I}(r)=C_{5} \operatorname{Re}\left[J_{n}\left(\sqrt{i k_{2} r}\right)\right]+C_{6} \operatorname{Im}\left[\sqrt{i k_{2} r}\right]$

where $k_{2}=\left(\lambda^{4}-k^{4}\right)^{\frac{1}{4}}$.

Substitution of Eq. (10) in Eq. (3) gives the following:

$w_{I I}(r, \theta)=\left[C_{5} \operatorname{Re}\left[J_{n}\left(\sqrt{i k_{2} r}\right)\right]+C_{6} \operatorname{Im}\left[\sqrt{i k_{2} r}\right]\right]$

$\cos (n \theta)$

For an elastically restrained circular plate, the boundary conditions at the edge of the plate in terms of rotational and translational stiffness are given by the following expressions:

$v_{r}(r, \theta)=-K_{T} w_{I}(r, \theta)$

$M_{r}(r, \theta)=K_{R} \frac{\partial w}{\partial r}(r, \theta)$

where the shearing force and bending moment as per KelvinKirchhofff theory are defined as follows:

$V_{r}=-D\left[\frac{\partial}{\partial r} \nabla^{2} w+(1-v) \frac{1}{r} \frac{\partial}{\partial \theta}\left(\frac{1}{r} \frac{\partial^{2} w}{\partial r \partial \theta}-\frac{1}{r^{2}} \frac{\partial w}{\partial \theta}\right)\right]$

$M_{r}=-D \cdot\left[\frac{\partial^{2} w}{\partial r^{2}}+v \cdot\left(\frac{1}{r} \frac{\partial w}{\partial r}+\frac{1}{r^{2}} \frac{\partial^{2} w}{\partial \theta^{2}}\right)\right]$

B.C. A : For a circulate plate with outer edge elastically restrained against rotation only, the Eqs. (12) and (13) become:

$$
\begin{aligned}
& v_{r}(r, \theta)=-K_{T} w_{I}(r, \theta) \\
& M_{r}(r, \theta)=0
\end{aligned}
$$

From Eqs.(12a) \& (14)

$\left[\begin{array}{l}\frac{\partial}{\partial r} \nabla^{2} w_{I}(r, \theta)+ \\ (1-v) \frac{1}{r} \frac{\partial}{\partial \theta}\left(\frac{1}{r} \frac{\partial^{2} w_{l}(r, \theta)}{\partial r \partial \theta^{2}}-\frac{1}{r^{2}} \frac{\partial w_{l}(r, \theta)}{\partial \theta}\right)\end{array}\right]=\frac{K_{T}}{D} w_{I}(r, \theta)$ 
From Eqs.(13a) \& (15):

$\left[\begin{array}{l}\frac{\partial^{2} w_{l}(r, \theta)}{\partial r^{2}}+ \\ v\left(\frac{1}{r} \frac{\partial w_{l}(r, \theta)}{\partial r}+\frac{1}{r^{2}} \frac{\partial^{2} w_{l}(r, \theta)}{\partial \theta^{2}}\right)\end{array}\right]=0$

The plate is continuous in terms of displacement, slope and moment at $r=b$. Therefore, the boundary conditions are:

$w_{I}(b)=w_{I I}(b)$

$w_{I}^{\prime}(b)=w_{I I}^{\prime}(b)$

$w_{I}^{\prime \prime}(b)=w_{I I}^{\prime \prime}(b)$

$w_{I}^{\prime \prime}(b)=w_{I I}^{\prime \prime}(b)$

Then for region I, from Eqs. (5) and (17), we get the following expression:

$\left[\frac{k^{2}}{4} J_{m 2}+\frac{k v}{2} J_{m 1}-\left(\frac{k^{2}}{2}+v n^{2}\right) J_{n}(k)\right] C_{1}$

$+\left[\frac{k^{2}}{4} Y_{n 2}+\frac{k v}{2} Y_{n 1}-\left(\frac{k^{2}}{2}+v n^{2}\right) Y_{n}(k)\right] C_{2}$

$+\left[\frac{k^{2}}{4} I_{p 2}+\frac{k v}{2} I_{p 1}+\left(\frac{k^{2}}{2}-v n^{2}\right) I_{n}(k)\right] C_{3}$

$+\left[\frac{k^{2}}{4} k_{q 2}-\frac{k v}{2} k_{q 1}+\left(\frac{k^{2}}{2}-v n^{2}\right) k_{n}(k)\right] C_{4}=0$

B.C. B: For a circulate plate with outer edge elastically restrained against translation only, Eqs. (12) and (13) become:

$v_{r}(r, \theta)=0$

$M_{r}(r, \theta)=K_{R} \frac{\partial w}{\partial r}(r, \theta)$

Then for region (I, $n)$ from Eqs. (5) and (16), we get the following expression:

$\left[\begin{array}{l}\frac{k^{3}}{8} J_{m 3}+\frac{k^{2}}{4} J_{m 2}-\frac{k}{2}\left(\frac{3}{4} k^{2}+n^{2}(2-v)+1\right) J_{m 1} \\ +\left(n^{2}(2-v)-\frac{k^{2}}{2}-\frac{K_{T}}{D}\right) J_{n}(k)\end{array}\right] C_{1}+$
$\left[\begin{array}{l}\frac{k^{3}}{8} Y_{n 3}+\frac{k^{2}}{4} Y_{n 2}-\frac{k}{2}\left(\frac{3}{4} k^{2}+n^{2}(2-v)+1\right) Y_{n 1} \\ +\left(n^{2}(2-v)-\frac{k^{2}}{2}-\frac{K_{T}}{D}\right) Y_{n}(k)\end{array}\right] C_{2}+$
$\left[\begin{array}{l}\frac{k^{3}}{8} I_{p 3}+\frac{k^{2}}{4} I_{p 2}+\frac{k}{2}\left(\frac{3}{4} k^{2}+n^{2}(-2+v)-1\right) I_{p 1} \\ +\left(n^{2}(2-v)+\frac{k^{2}}{2}-\frac{K_{T}}{D}\right) I_{n}(k)\end{array}\right] C_{3}+$
$\left[\begin{array}{l}\frac{k^{3}}{8} k_{Q 3}+\frac{k^{2}}{4} K_{Q 2}+\frac{k}{2}\left(-\frac{3}{4} k^{2}+n^{2}(2-v)+1\right) K_{Q 1} \\ +\left(n^{2}(2-v)+\frac{k^{2}}{2}-\frac{K_{T}}{D}\right) K_{n}(k)\end{array}\right] C_{4}=0$

where
$J_{m 1}=J_{n-1}(k)-J_{n+1}(k) ; J_{m 2}=J_{n-2}(k)+J_{n+2}(k) ;$

$J_{m 3}=J_{n-3}(k)-J_{n+3}(k) ; Y_{n 1}=Y_{n-1}(k)-Y_{n+1}(k)$;

$Y_{n 2}=Y_{n-2}(k)+Y_{n+2}(k) ; Y_{n 3}=Y_{n-3}(k)-Y_{n+3}(k) ;$

$I_{p 1}=I_{n-1}(k)+I_{n+1}(k) ; I_{p 2}=I_{n-2}(k)+I_{n+2}(k) ;$

$I_{p 3}=I_{n-3}(k)+I_{n+3}(k) ; K_{q 1}=K_{n-1}(k)+K_{n+1}(k)$;

$K_{q 2}=K_{n-2}(k)+K_{n+2}(k) ; K_{q 3}=K_{n-3}(k)+K_{n+3}(k)$;

Case (i): Considering the case (i) i.e. $k>\lambda$, for the region (II, $n$ ), the solution to Eq. (2) is given by Eq.(7) and for this case the boundary conditions, Eqs. (16), (17), (18) and (19) give us the following:

$J_{n}(k b) C_{1}+Y_{n}(k b) C_{2}+I_{n}(k b) C_{3}+K_{n}(k b) C_{4}$

$-J_{n}(k 1 b) C_{5}-I_{n}(k 1 b) C_{6}=0$

$\frac{k}{2} J_{m 1}^{\prime} C_{1}+\frac{k}{2} Y_{n 1}^{\prime} C_{2}+\frac{k}{2} I_{p 1}^{\prime} C_{3}-\frac{k}{2} K_{q 1}^{\prime} C_{4}-$

$\frac{k_{1}}{2} J_{m 11}^{\prime} C_{5}-\frac{k}{2} I_{p 11}^{\prime} C_{6}=0$

$\frac{k^{2}}{4}\left(J_{m 2}^{\prime}-2 J_{n}(k b)\right) C_{1}+\frac{k^{2}}{4}\left(Y_{n 2}^{\prime}-2 Y_{n}(k b)\right) C_{2}+$

$\frac{k^{2}}{4}\left(I_{p 2}^{\prime}+2 I_{n}(k b)\right) C_{3}+\frac{k^{2}}{4}\left(K_{q 2}^{\prime}+2 K_{n}(k b)\right) C_{4}$

$-\frac{k_{1}^{2}}{4}\left(J_{m 22}^{\prime}-2 J_{n}\left(k_{1} b\right)\right) C_{5}-$

$\frac{k_{1}^{2}}{4}\left(I_{p 22}^{\prime}+2 I_{n}\left(k_{1} b\right)\right) C_{6}=0$

$\frac{k^{3}}{8}\left[J_{m 3}^{\prime}-3 J_{m 1}^{\prime}\right] C_{1}+\frac{k^{3}}{8}\left[Y_{n 3}^{\prime}-3 Y_{n 1}^{\prime}\right] C_{2}+$

$\frac{k^{3}}{8}\left[I_{p 3}^{\prime}+3 I_{p 1}^{\prime}\right] C_{3}-\frac{k^{3}}{8}\left[K_{q 3}^{\prime}+3 K_{q 1}^{\prime}\right] C_{4}-$

$\frac{k_{1}^{3}}{8}\left[J_{m 33}^{\prime}-3 J_{m 11}^{\prime}\right] C_{5}-$

$\frac{k_{1}^{3}}{8}\left[I_{p 33}^{\prime}+3 I_{p 11}^{\prime}\right] C_{6}=0$

Case (ii): Considering the case (ii) i.e. $k=\lambda$, for the region (II, $n$ ), the solution to Eq. (2) is given by Eq. (9) and for this case the boundary conditions, Eqs. (16), (17), (18) and (19) give us the following:

$J_{n}(k b) C_{1}+Y_{n}(k b) C_{2}+I_{n}(k b) C_{3}$

$+K_{n}(k b) C_{4}-b^{n} C_{5}-b^{n+2} C_{6}=0$

$\frac{k}{2} J_{m 1}^{\prime} C_{1}+\frac{k}{2} Y_{n 1}^{\prime} C_{2}+\frac{k}{2} I_{p 1}^{\prime} C_{3}-$

$\frac{k}{2} K_{q 1}^{\prime} C_{4}-n b^{n-1} C_{5}-(n+2) b^{n+1} C_{6}=0$

$\frac{k^{2}}{4}\left(J_{m 2}^{\prime}-2 J_{n}(k b)\right) C_{1}+\frac{k^{2}}{4}\left(Y_{n 2}^{\prime}-2 Y_{n}(k b)\right) C_{2}$

$+\frac{k^{2}}{4}\left(I_{p 2}^{\prime}+2 I_{n}(k b)\right) C_{3}+\frac{k^{2}}{4}\left(K_{q 2}^{\prime}+2 K_{n}(k b)\right) C_{4}$

$-\left(n(n-1) b^{n-2}\right) C_{5}-(n+1)(n+2) b^{n} C_{6}=0$

$\frac{k^{3}}{8}\left[J_{m 3}^{\prime}-3 J_{m 1}^{\prime}\right] C_{1}+\frac{k^{3}}{8}\left[Y_{n 3}^{\prime}-3 Y_{n 1}^{\prime}\right] C_{2}$

$+\frac{k^{3}}{8}\left[I_{p 3}^{\prime}+3 I_{p 1}^{\prime}\right] C_{3}-\frac{k^{3}}{8}\left[K_{q 3}^{\prime}+3 K_{q 1}^{\prime}\right] C_{4}-$

$\left[n(n-1)(n-2) b^{n-3}\right] C_{5}-\left[n(n+1)(n+2) b^{n-1}\right] C_{6}=0$

where 
$J_{m 1}^{\prime}=J_{n-1}(k b)-J_{n+1}(k b) ; J_{m 2}^{\prime}=J_{n-2}(k b)+J_{n+2}(k b) ;$

$J_{m 3}^{\prime}=J_{n-3}(k b)-J_{n+3}(k b) Y_{n 1}^{\prime}=Y_{n-1}(k b)-Y_{n+1}(k b)$;

$Y_{n 2}^{\prime}=Y_{n-2}(k b)+Y_{n+2}(k b) ; Y_{n 3}^{\prime}=Y_{n-3}(k b)-Y_{n+3}(k b)$

$I_{p 1}^{\prime}=I_{n-1}(k b)+I_{n+1}(k b) ; I_{p 2}^{\prime}=I_{n-2}(k b)+I_{n+2}(k b)$;

$I_{p 3}^{\prime}=I_{n-3}(k b)+I_{n+3}(k b) K_{q 1}^{\prime}=K_{n-1}(k b)+K_{n+1}(k b)$;

$K_{q 2}^{\prime}=K_{n-2}(k b)+K_{n+2}(k b) ; K_{q 3}^{\prime}=K_{n-3}(k b)+K_{n+3}(k b)$

Case (iii) : Considering the case (iii), for $k<\lambda$, for region (II, $n$ ), the solution to Eq. (2) is given by Eq. (11) and for this case the boundary conditions Eqs. (16), (17), (18) and (19) give us the following:

$J_{n}(k b) C_{1}+Y_{n}(k b) C_{2}+I_{n}(k b) C_{3}+K_{n}(k b) C_{4}$

$-\operatorname{Re}\left[J_{n}\left(\sqrt{i k_{2} b}\right)\right] C_{5}-\operatorname{Im}\left[J_{n}\left(\sqrt{i k_{2} b}\right)\right] C_{6}=0$

$J_{n}^{\prime}(k b) C_{1}+Y_{n}^{\prime}(k b) C_{2}+I_{n}^{\prime}(k b) C_{3}-K_{n}^{\prime}(k b) C_{4}-$

$\operatorname{Re}\left[J_{m 1}^{\prime}\left(\sqrt{i k_{2} b}\right)\right] C_{5}-\operatorname{Im}\left[J_{m 1}^{\prime}\left(\sqrt{i k_{2} b}\right)\right] C_{6}=0$

$J_{n}^{\prime \prime}(k b) C_{1}+Y_{n}^{\prime \prime}(k b) C_{2}+I^{\prime \prime}{ }_{n}(k b) C_{3}-K^{\prime \prime}{ }_{n}(k b) C_{4}-$

$\operatorname{Re}\left[J^{\prime \prime}{ }_{m 1}\left(\sqrt{i k_{2} b}\right)\right] C_{5}-\operatorname{Im}\left[J^{\prime \prime}{ }_{m 1}\left(\sqrt{i k_{2} b}\right)\right] C_{6}=0$

$J{ }^{\prime \prime}{ }_{n}(k b) C_{1}+Y^{\prime \prime \prime}{ }_{n}^{\prime}(k b) C_{2}+I^{\prime \prime}{ }_{n}(k b) C_{3}-K^{\prime \prime}{ }_{n}(k b) C_{4}-$

$\operatorname{Re}\left[J^{\prime \prime \prime}{ }_{m 1}\left(\sqrt{i k_{2} b}\right)\right] C_{5}-\operatorname{Im}\left[J^{\prime \prime}{ }_{m 1}\left(\sqrt{i k_{2} b}\right)\right] C_{6}=0$

Therefore, the set of Eqs. (20), (21), (30)-(33), represent for the case $k<\lambda$.

\section{SOLUTION}

For the given values of $n, v, b, T_{11}, R_{11} \& \lambda$ the above set of equations gives an exact characteristic equation for nontrivial solutions of the coefficients $C_{1}, C_{2}, C_{3}, C_{4}, C_{5} \& C_{6}$. For non-trivial solution, the determinant of $[C]_{6 \times 6}$ must vanish. This eigenvalue problem was solved using Mathematica computer software with symbolic capabilities.

\section{RESULTS \& DISCUSSIONS}

There is a lot of flexibility in the code developed in Mathematica. It is used to determine the frequency parameter for any range of translational constraints. This code is also implanted for various plate materials by adjusting Poisson's ratio. Since Poisson's ratio occurs as a parameter in most of the equations, the effect of this ratio on the roots of the equations is also considered. The findings are presented in both tabular and graphical form. The frequencies are calculated for various radius radii $b$, translational spring stiffness parameter.

The frequency values for the plate with elastically restrained edge against translation and resting on partial foundation, at various values of the translational stiffness parameter, $T_{11}$ and for constant $\lambda$, have been calculated and the results are shown in the Table $\mathbf{1}$ and graphically in Fig. (2). The frequency has increased considerably as increase in translational constraint. The results also listed in Table $\mathbf{2}$ and graphically in Fig. (3), for different values of foundation constraint $(\lambda)$ by keeping translational constraint $\left(T_{11}\right)$ constant. For large foundation stiffness the curves will be asymptotic in nature. The results are shown in Fig. (4) for different values of Translational and foundation constraints. It is observed that the frequency is increases as two constraints increase simultaneously. It was found that the $n=0$ axisymmetric mode gives the fundamental frequency. When $b=0$, the foundation is absent and the frequency is governed by the elastically restrained edge plate, i.e. $k=$ 2.1834. When $b=1$, the plate has full foundation support, and the frequency is $\mathrm{k}_{0}=2.1834$. The effect of Translational constraint and Foundation constraint on frequency is shown in Figs. $(2,3)$ respectively. Also the combined effect of translational and Foundation constraints are shown in Fig. (4).

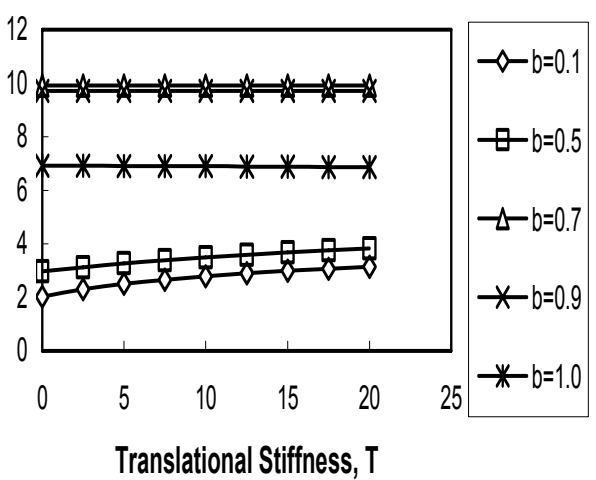

Fig. (2). Effect of translational stiffness parameter, $T_{11}$ on first natural frequency parameter, $k$ for $\lambda=10$.

Table 1. First Frequency Parameters for Different Translational Stiffness Ratio for $\lambda=10 \& v=0.33$

\begin{tabular}{|c|c|c|c|c|c|c|c|c|c|c|}
\hline $\boldsymbol{T}_{\mathbf{1 1}}$ & $\boldsymbol{b}=\mathbf{0 . 1}$ & $\boldsymbol{b}=\mathbf{0 . 2}$ & $\boldsymbol{b}=\mathbf{0 . 3}$ & $\boldsymbol{b}=\mathbf{0 . 4}$ & $\boldsymbol{b}=\mathbf{0 . 5}$ & $\boldsymbol{b}=\mathbf{0 . 6}$ & $\boldsymbol{b}=\mathbf{0 . 7}$ & $\boldsymbol{b}=\mathbf{0 . 8}$ & $\boldsymbol{b}=\mathbf{0 . 9}$ & $\boldsymbol{b}=\mathbf{1}$ \\
\hline \hline 0 & 2.02235 & 2.00269 & 1.65954 & 3.17252 & 2.97074 & 2.59295 & 9.91088 & 9.82665 & 9.71751 & 6.9251 \\
\hline 2.5 & 2.30653 & 2.28733 & 2.06005 & 3.32198 & 3.1293 & 2.81866 & 9.91072 & 9.82583 & 9.71713 & 6.91746 \\
\hline 5 & 2.50643 & 2.48571 & 2.29898 & 3.45341 & 3.26574 & 2.99905 & 9.91056 & 9.82492 & 9.71666 & 6.90972 \\
\hline 7.5 & 2.66201 & 2.63915 & 2.47235 & 3.5711 & 3.38604 & 3.15054 & 9.91039 & 9.824 & 9.71627 & 6.90198 \\
\hline 10 & 2.78959 & 2.7643 & 2.60887 & 3.67793 & 3.49379 & 3.28191 & 9.91023 & 9.82319 & 9.71579 & 6.89404 \\
\hline 12.5 & 2.89784 & 2.86973 & 2.72118 & 3.77599 & 3.59168 & 3.39823 & 9.91007 & 9.82227 & 9.71541 & 6.8862 \\
\hline 15 & 2.99165 & 2.96052 & 2.81616 & 3.86678 & 3.68139 & 3.5029 & 9.9099 & 9.82135 & 9.71492 & 6.87817 \\
\hline 17.5 & 3.07421 & 3.03995 & 2.89819 & 3.95139 & 3.76433 & 3.5981 & 9.90974 & 9.82044 & 9.71444 & 6.87013 \\
\hline 20 & 3.1477 & 3.11033 & 2.96997 & 4.03073 & 3.84139 & 3.68573 & 9.90957 & 9.81952 & 9.71404 & 6.86199 \\
\hline
\end{tabular}


Table 2. First Frequency for Different Foundation Stiffness Parameter for $T_{11} \& v=0.33$

\begin{tabular}{|c|c|c|c|c|c|c|c|c|c|c|}
\hline $\boldsymbol{\lambda}$ & $\boldsymbol{b}=\mathbf{0 . 1}$ & $\boldsymbol{b}=\mathbf{0 . 2}$ & $\boldsymbol{b}=\mathbf{0 . 3}$ & $\boldsymbol{b}=\mathbf{0 . 4}$ & $\boldsymbol{b}=\mathbf{0 . 5}$ & $\boldsymbol{b}=\mathbf{0 . 6}$ & $\boldsymbol{b}=\mathbf{0 . 7}$ & $\boldsymbol{b}=\mathbf{0 . 8}$ & $\boldsymbol{b}=\mathbf{0 . 9}$ & $\boldsymbol{b}=\mathbf{1}$ \\
\hline \hline 0 & 1.85759 & 1.85759 & 1.85759 & 1.85759 & 1.85759 & 1.85759 & 1.85759 & 1.85759 & 1.85759 & 1.85759 \\
\hline 7.5 & 2.95671 & 2.82178 & 2.81512 & 2.66589 & 6.00336 & 4.8109 & 4.54729 & 4.3199 & 6.40789 & 6.36732 \\
\hline 10 & 2.78959 & 2.7643 & 2.60887 & 3.67793 & 3.49379 & 3.28191 & 9.91023 & 9.82319 & 9.71579 & 6.89404 \\
\hline 12.5 & 2.74279 & 2.72015 & 3.46242 & 3.2138 & 7.75637 & 4.58806 & 4.45195 & 11.6979 & 11.5023 & 11.3633 \\
\hline 15 & 2.72551 & 2.58 & 3.0792 & 2.94435 & 3.83438 & 3.65997 & 6.43791 & 5.90339 & 12.7186 & 12.6973 \\
\hline 17.5 & 2.71662 & 3.36935 & 2.9918 & 3.62631 & 3.4595 & 4.69047 & 4.25264 & 8.95145 & 12.9626 & 13.52 \\
\hline 20 & 2.70984 & 2.96343 & 2.83938 & 3.31891 & 4.39574 & 3.9389 & 5.6791 & 18.1682 & 17.9198 & 13.6294 \\
\hline
\end{tabular}

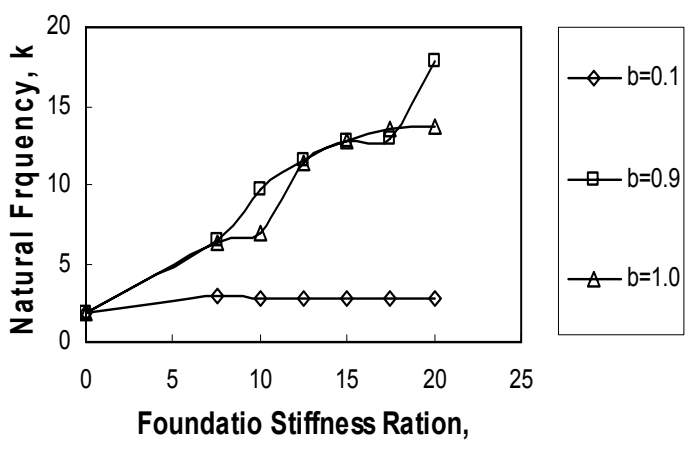

Fig. (3). Effect of foundation stiffness parameter, $\lambda$ on first natural frequency parameter, $k$ for $T_{11}=10$.

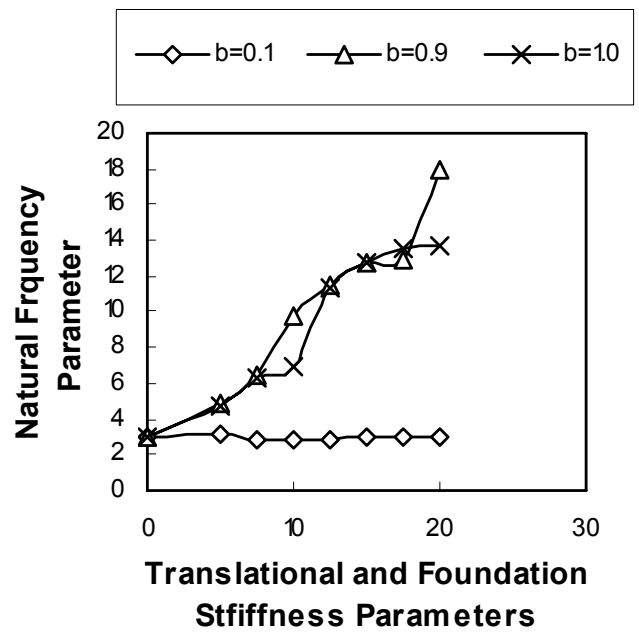

Fig. (4). Effect of translational stiffness parameter, $T_{11}$ and foundation stiffness parameter $\lambda$ on natural frequency parameter, $k$.

The frequencies for different plate materials, for various values of transverse, rotational and foundation parameters are computed and the results are given in Table $\mathbf{3}$. It is observed that for any value of foundation parameter $(\lambda)$, frequencies are independent on Poisson ratio, as shown in
Fig. (5). And also it was observed that for any value of $T_{11}$, frequencies are independent on Poisson ratio.

Table 3. Frequencies for Different Poisson Ratios

\begin{tabular}{|c|c|c|c|}
\hline $\boldsymbol{v}$ & $\mathbf{T}_{\mathbf{1 1}}=\mathbf{1 0}, \boldsymbol{\lambda}=\mathbf{1 0}$ & $\mathbf{T}_{\mathbf{1 1}}=\mathbf{1 0 0 0}, \boldsymbol{\lambda}=\mathbf{1 0}$ & $\mathbf{T}_{\mathbf{1 1}}=\mathbf{1 0}, \boldsymbol{\lambda}=\mathbf{1 0 0 0}$ \\
\hline \hline 0 & 2.77951 & 4.37181 & 2.78038 \\
\hline 0.1 & 2.7831 & 4.39014 & 2.78338 \\
\hline 0.2 & 2.7865 & 4.40768 & 2.78608 \\
\hline 0.3 & 2.78959 & 4.42472 & 2.78857 \\
\hline 0.4 & 2.79248 & 4.44116 & 2.79087 \\
\hline 0.5 & 2.79517 & 4.4571 & 2.79306 \\
\hline
\end{tabular}

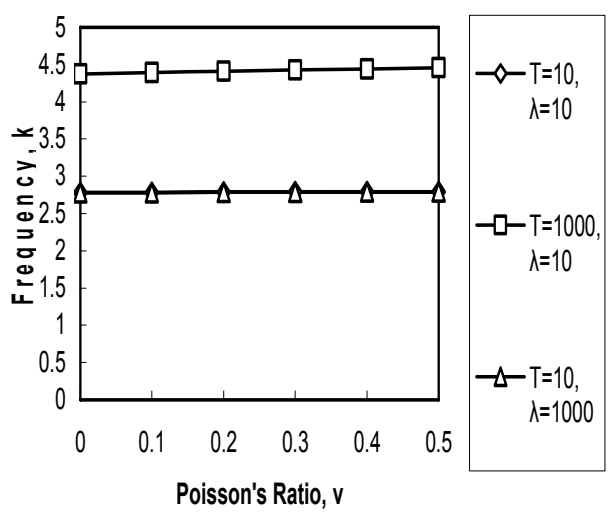

Fig. (5). Effect of poisson ratio, ${ }_{v}$ on frequency parameters, $k$.

The results of this kind are scarce in the literature. However, the results are compared with the following. If $R_{11} \rightarrow 0 \& T_{11} \rightarrow \infty$, then the problem at hand becomes a simply supported boundary condition as shown in Fig. (6). The results are listed in Table 4. It was found that the $n=0$ axisymmetric mode gives the fundamental frequency. When $b=0$, the foundation is absent and the circular simply supported plate governs the frequency, i.e., $k=2.22152$. When $b=1$, the plate has full foundation support and the frequency is $k_{0}=2.22152$. Table $\mathbf{5}$, presents the comparison of frequency parameters $k$, for the plate with simply 
supported edges as shown in Fig. (6) (by setting the translational restraints with $\left.R_{11} \rightarrow 0 \& T_{11} \rightarrow \infty\right)$, against those obtained by Wang [13] and Laura et al [6] by Ritz and finite element methods respectively. Results presented in this paper can be seen to be in excellent agreement with the those results available in the literature.

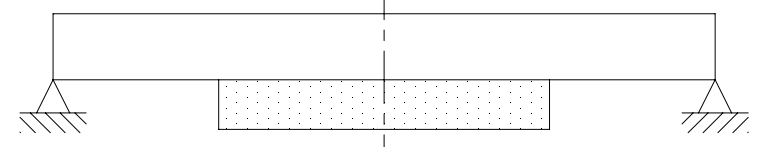

Fig. (6). A thin circular plate with simply supported edge and supported on partial elastic foundation.

Table 4. Frequency for Different Foundation Stiffness Ratio for $v=\mathbf{0 . 3 3}$

\begin{tabular}{|c|c|c|c|c|}
\hline $\boldsymbol{\lambda}$ & $\boldsymbol{b}=\mathbf{0 . 1}$ & $\boldsymbol{b}=\mathbf{0 . 3}$ & $\boldsymbol{b}=\mathbf{0 . 4}$ & $\boldsymbol{b}=\mathbf{0 . 9}$ \\
\hline \hline 0 & 2.2215 & 2.2215 & 2.2215 & 2.22152 \\
\hline 20 & 3.9522 & 4.5808 & 6.0081 & 14.6284 \\
\hline 50 & 4.1065 & 5.1786 & 6.336 & 29.1056 \\
\hline 100 & 4.2517 & 5.3877 & 6.228 & 36.7841 \\
\hline 500 & 4.1747 & 5.4437 & 6.3845 & 39.3756 \\
\hline 1000 & 4.2049 & 5.4673 & 6.4141 & 38.8204 \\
\hline 2000 & 4.214 & 5.3866 & 6.41 & 37.7465 \\
\hline 5000 & 4.2186 & 5.4738 & 6.4151 & 39.1173 \\
\hline 7500 & 4.2165 & 5.4756 & 6.4103 & 39.1006 \\
\hline 10000 & 4.2176 & 5.4708 & 6.4183 & 39.0791 \\
\hline
\end{tabular}

Table 5. Comparison of Exact Values with Approximate Values from Ref. [6, 13, 14] for Simply Supported Edge Plate

\begin{tabular}{|l|c|c|c|c|}
\hline $\mathbf{b}$ & 0.3 & 0.3 & 0.6 & 0.6 \\
\hline $\boldsymbol{\lambda}$ & 2.1147 & 3.1623 & 2.1147 & 3.1623 \\
\hline $\boldsymbol{k}$ [Present] & 2.33844 & 2.67264 & 2.51384 & 3.17202 \\
\hline $\boldsymbol{k}[\mathbf{1 2}]$ & 2.33844 & 2.67274 & 2.51304 & 3.17204 \\
\hline Ritz [6] & 2.339 & 2.677 & 2.514 & 3.1724 \\
\hline F.E [6] & 2.349 & 2.702 & 2.536 & 3.2249 \\
\hline $\boldsymbol{k}[\mathbf{1 3}]$ & 2.33844 & 2.67264 & 2.51384 & 3.17202 \\
\hline
\end{tabular}

* The results are approximate.

If $R_{11} \rightarrow \infty \& T_{11} \rightarrow \infty$, then the problem at hand becomes a clamped boundary condition as shown in Fig. (7). The results are listed in Table 6. It was found that the $n=0$ axisymmetric mode gives the fundamental frequency. When $b=0$, the foundation is absent and the circular clamped plate governs frequency, i.e., $k=3.19622$.

When $b=1$, the plate has full foundation support and the frequency is $k_{0}=3.19622$. Table 7 shows a comparison of our exact values with the values obtained by Wang [13] and
Laura et al. [6] by Ritz and finite element methods respectively. Excellent agreement has been found between these results.

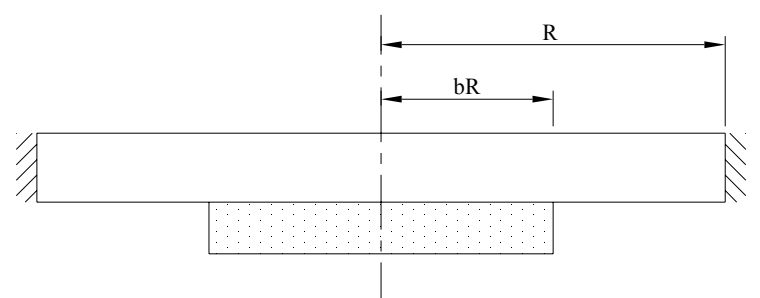

Fig. (7). A thin circular plate with clamped edge and supported on partial elastic foundation.

Table 6. Frequency for Different Foundation Stiffness Ratio for $v=0.33$

\begin{tabular}{|c|c|c|c|c|}
\hline $\boldsymbol{\lambda}$ & $\boldsymbol{b}=\mathbf{0 . 3}$ & $\boldsymbol{b}=\mathbf{0 . 4}$ & $\boldsymbol{b}=\mathbf{0 . 8}$ & $\boldsymbol{b}=\mathbf{1 . 0}$ \\
\hline \hline 0 & 3.19622 & 3.19622 & 3.1962 & 3.19622 \\
\hline 20 & 5.73303 & 7.42844 & 18.151 & 13.536 \\
\hline 50 & 6.38558 & 7.77718 & 21.658 & 33.8797 \\
\hline 100 & 6.63007 & 7.64012 & 22.987 & 56.2845 \\
\hline 500 & 6.69625 & 7.82554 & 23.572 & 124.841 \\
\hline 1000 & 6.72454 & 7.86099 & 23.474 & 249.707 \\
\hline 2000 & 6.62889 & 7.85603 & 23.633 & 499.426 \\
\hline 5000 & 6.73224 & 7.86222 & 23.649 & 1002.67 \\
\hline 10000 & 6.72868 & 7.86598 & 23.636 & 1200.98 \\
\hline 20000 & 6.73245 & 7.86527 & 23.597 & 1600.78 \\
\hline
\end{tabular}

Table 7. Comparison of Exact Values with Approximate Values from Ref. [6, 12] for Clamped Edge Plate

\begin{tabular}{|c|c|c|c|c|}
\hline $\boldsymbol{b}$ & 0.3 & 0.3 & 0.6 & 0.6 \\
\hline $\boldsymbol{\lambda}$ & 2.1147 & 3.1623 & 2.1147 & 3.1623 \\
\hline $\boldsymbol{k}$ [Present] & 3.25572 & 3.46125 & 3.32705 & 3.73673 \\
\hline $\boldsymbol{k}$ [12] & 3.25573 & 3.46124 & 3.32706 & 3.73672 \\
\hline Ritz [6] ${ }^{*}$ & 3.2558 & 3.4615 & 3.3275 & 3.7367 \\
\hline F.E [6] & 3.2558 & 3.4771 & 3.1237 & 3.6576 \\
\hline
\end{tabular}

\section{CONCLUSIONS}

The flexural vibration behaviour of a circular plate supported along its edge by elastically restrained springs against rotation and translational and supported partially on a Winkler-type foundation has been studied in this paper. Mathematica computer software was used in obtaining the results for first frequency values of this circular plate.

The values of first frequencies are presented in both tabular and graphical form for various values of translational spring stiffness parameters $\left[R_{11} \& T_{11}\right]$ at the edges that 
simulate a clamped edge when $R_{11} \rightarrow \infty \& T_{11} \rightarrow \infty$, or a simply supported edge when $R_{11} \rightarrow 0 \& T_{11} \rightarrow \infty$.

Graphical plots of first frequencies are presented for a wide range of rotational, translational and foundation constraints. The wide range of results provided in this paper could be potentially utilized for vibration control and in structural design. It is observed that the influence of foundation parameter on frequency is more predominant than that of translational parameter or rotational parameter.

Comparison of results obtained here with those available in literature for some special cases, demonstrates excellent accuracy and numerical stability of the present method. In this paper the characteristic equations solved are exact ones and therefore the frequency results can be calculated to any desired accuracy. These exact solutions can be used as benchmark solutions to check numerical or approximate results obtained through other methods of solution.

\section{NOTATIONS}

$$
\begin{array}{ll}
h & =\text { Thickness of a plate } \\
R & =\text { Radius of a plate } \\
b & =\text { Non-dimensional radius of support } \\
v & =\text { Poisson's ratio } \\
E & =\text { Young's modulus of a material } \\
\rho & =\text { Density of a material } \\
\omega & =\text { Angular frequency } \\
D & =\text { Flexural rigidity of a material } \\
K_{R 1} & =\text { Rotational spring stiffness at outer edge } \\
K_{T 2} & =\text { Translational spring stiffness at outer edge }
\end{array}
$$

$$
\begin{aligned}
& R_{11} \quad=\text { Non-dimensional rotational flexibility } \\
& \text { Parameter at outer edge } \\
& T_{22} \quad=\text { Non-dimensional translational flexibility } \\
& \text { Parameter at outer edge } \\
& \lambda=\text { Non-dimensional foundation parameter }
\end{aligned}
$$

\section{REFERENCES}

[1] Leissa AW. Plate vibration research: 1976-1980: complicating effects. Shock Vibration Digest 1981; 13(10): 19-36.

[2] Leissa AW. Resent research in plate vibrations: 1981-1985 Part II: complicating effects. Shock Vibrations Digest 1987; 19(3): 10-24.

[3] Bert CW. Research on dynamic behavior of composite and sandwich plates-IV. Shock Vibration Digest 1985; 17(11): 3-25.

[4] Bert CW. Research on dynamic behavior of composite and sandwich plates-V: Part I. Shock Vibration Digest 1991; 23(6): 314.

[5] Leissa AW. Vibration of Plates, Acoustical Society of America, Sewickley. P. A. 1993.

[6] Laura PAA, Gutierrez RH, Sanzi HC, Elvira G. The lowest axisymmetrical frequency of vibration of a circular plate partially embedded in a Winkler foundation. J Sound Vibration 1995; 185: 915-9.

[7] Winkler E. Die Lehre von der Elasticitaet and Festigkeit, Prag. Dominicus 1867 .

[8] Dumir PC. Large deflection axisymmetric analysis of orthotropic annular plates on elastic Foundations. Int J Solids Struct 1988; 24 : 777-87.

[9] Bhaskara Rao L. Study of Static Stability of Circular and Annular Plates on Generalized Elastic Medium, PhD Thesis, Osmania University, Hyderabad, A.P. India 2008

[10] Kim CS, Dickinson SM. The flexural vibration of thin isotropic and polar orthtropic annular and circular plates with elastically restrained peripheries. J Sound Vibration 1990; 143: 171-9.

[11] Amabili M, Pierandrei R. Analysis of vibrating circular plates having non-uniform constraint using the modal properties of free edge plates: Application to bolted plates. J Sound Vibration 1997; 206 (1): $23-38$

[12] Yu SC, Huang SC. Vibration of three-layered viscoelastic sandwich circular plate. Int J Mech Sci 2001; 43: 2215-36.

[13] Wang CM. Fundamental frequency of a circular plate supported by a partial elastic foundation. J Sound Vibration 2005; 285: 1203-9. 